\title{
The Peak of Noncentral Stirling Numbers of the First Kind
}

\author{
Roberto B. Corcino, ${ }^{1}$ Cristina B. Corcino, ${ }^{1}$ and Peter John B. Aranas ${ }^{2}$ \\ ${ }^{1}$ Mathematics and ICT Department, Cebu Normal University, 6000 Cebu City, Philippines \\ ${ }^{2}$ Department of Mathematics, Mindanao State University, Main Campus, 9700 Marawi City, Philippines
}

Correspondence should be addressed to Roberto B. Corcino; rcorcino@yahoo.com

Received 18 September 2014; Accepted 20 November 2014

Academic Editor: Serkan Araci

Copyright (C) 2015 Roberto B. Corcino et al. This is an open access article distributed under the Creative Commons Attribution License, which permits unrestricted use, distribution, and reproduction in any medium, provided the original work is properly cited.

We locate the peak of the distribution of noncentral Stirling numbers of the first kind by determining the value of the index corresponding to the maximum value of the distribution.

\section{Introduction}

In 1982, Koutras [1] introduced the noncentral Stirling numbers of the first and second kind as a natural extension of the definition of the classical Stirling numbers, namely, the expression of the factorial $(x)_{n}$ in terms of powers of $x$ and vice versa. These numbers are, respectively, denoted by $s_{a}(n, k)$ and $S_{a}(n, k)$ which are defined by means of the following inverse relations:

$$
\begin{gathered}
(t)_{n}=\sum_{k=0}^{n} \frac{1}{k !}\left[\frac{d^{k}}{d t^{k}}(t)_{v}\right]_{t=a}(t-a)^{k}, \\
(t-a)^{n}=\sum_{k=0}^{n} \frac{1}{k !}\left[\Delta^{k}(t-a)^{n}\right]_{t=0}(t)_{k},
\end{gathered}
$$

where $a, t$ are any real numbers, $n$ is a nonnegative integer, and

$$
\begin{aligned}
& s_{a}(n, k)=\frac{1}{k !}\left[\frac{d^{k}}{d t^{k}}(t)_{v}\right]_{t=a}, \\
& S_{a}(n, k)=\frac{1}{k !}\left[\Delta^{k}(t-a)^{n}\right]_{t=0} .
\end{aligned}
$$

The numbers satisfy the following recurrence relations:

$$
\begin{aligned}
& s_{a}(n+1, k)=s_{a}(n, k-1)+(a-n) s_{a}(n, k), \\
& S_{a}(n+1, k)=S_{a}(n, k-1)+(k-a) S_{a}(n, k)
\end{aligned}
$$

and have initial conditions

$$
\begin{gathered}
s_{a}(0,0)=1, \quad s_{a}(n, 0)=(a)_{n}, \quad s_{a}(0, k)=0, \quad n, k \neq 0, \\
S_{a}(0,0)=1, \quad S_{a}(n, 0)=(-a)^{n}, \quad S_{a}(0, k)=0, \quad n, k \neq 0 .
\end{gathered}
$$

It is worth mentioning that for a given negative binomial distribution $Y$ and the sum $X=X_{1}+X_{2}+\cdots+X_{k}$ of $k$ independent random variables following the logarithmic distribution, the numbers $s_{a}(n, k)$ appeared in the distribution of the sum $W=X+Y$, while the numbers $S_{a}(n, k)$ appeared in the distribution of the sum $Z=\widehat{X}+\widehat{Y}$ where $\widehat{X}$ is the sum of $k$ independent random variables following the truncated Poisson distribution away from zero and $\hat{Y}$ is a Poisson random variable. More precisely, the probability distributions of $W$ and $Z$ are given, respectively, by

$$
\begin{aligned}
& P[W=n]=\frac{k !}{(1-\theta)^{-s}(-\log (1-\theta))^{k}} \frac{\theta^{n}}{n !}(-1)^{n-k} s_{-s}(n, k), \\
& P[Z=n]=\frac{k !}{e^{m}\left(e^{l}-1\right)^{k}} \frac{l^{n}}{n !}(-1)^{n-k} S_{-m / l}(n, k) .
\end{aligned}
$$

For a more detailed discussion of noncentral Stirling numbers, one may see [1].

Determining the location of the maximum of Stirling numbers is an interesting problem to consider. In [2], Mezö 
obtained results for the so-called $r$-Stirling numbers which are natural generalizations of Stirling numbers. He showed that the sequences of $r$-Stirling numbers of the first and second kinds are strictly log-concave. Using the theorem of Erdös and Stone [3] he was able to establish that the largest index for which the sequence of $r$-Stirling numbers of the first kind assumes its maximum is given by the approximation

$$
K_{n, r}^{(1)}=r+\left[\log \left(\frac{n-1}{r-1}\right)-\frac{1}{r}+o(1)\right] .
$$

Following the methods of Mezö, we establish strict logconcavity and hence unimodality of the sequence of noncentral Stirling numbers of the first kind and, eventually, obtain an estimating index at which the maximum element of the sequence of noncentral Stirling numbers of the first kind occurs.

\section{Explicit Formula}

In this section, we establish an explicit formula in symmetric function form which is necessary in locating the maximum of noncentral Stirling numbers of the first kind.

Let $f_{i}(x), i=1,2, \ldots, n$ be differentiable functions and let $F_{n}(x)=\prod_{i=1}^{n} f_{i}(x)$. It can easily be verified that, for all $n \geq 3$,

$$
F_{n}^{\prime}(x)=\sum_{\substack{1 \leq j_{1}<j_{2}<\cdots<j_{n-1} \leq n \\ i \in \mathbb{N}_{n} \backslash\left\{j_{1}, j_{2}, \ldots, j_{n-1}\right\}}} f_{i}^{\prime}(x) \prod_{k=1}^{n-1} f_{j_{k}}(x) .
$$

Now, consider the following derivative of $(\xi j+a)_{n}$ when $n=$ 1,2 :

$$
\begin{gathered}
\frac{d}{d \xi}(\xi j+a)_{1}=j, \\
\frac{d}{d \xi}(\xi j+a)_{2}=(\xi j+a) j+(\xi j+a-1) j .
\end{gathered}
$$

Then, for $n \geq 3$ and using (9), we get

$$
\frac{1}{j^{n}} \frac{d}{d \xi}(\xi j+a)_{n}=\sum_{0 \leq j_{1}<j_{2}<\cdots<j_{n-1} \leq n-1} \prod_{k=1}^{n-1}\left(\xi+\frac{a-j_{k}}{j}\right) .
$$

Then, we have the following lemma.

Lemma 1. For any nonnegative integers $n$ and $k$, one has

$$
\frac{1}{j^{n}} \frac{d^{k}}{d \xi^{k}}(\xi j+a)_{n}=\sum_{0 \leq j_{1}<j_{2}<\cdots<j_{n-k} \leq n-1} k ! \prod_{q=1}^{n-k}\left(\xi+\frac{a-j_{q}}{j}\right) .
$$

Proof. We prove by induction on $k$. For $k=0$, (12) clearly holds. For $k=1,(12)$ can easily be verified using (11). Suppose for $m \geq 1$,

$$
\frac{1}{j^{n}} \frac{d^{m}}{d \xi^{m}}(\xi j+a)_{n}=\sum_{0 \leq j_{1}<j_{2}<\cdots<j_{n-m} \leq n-1} m ! \prod_{q=1}^{n-m}\left(\xi+\frac{a-j_{q}}{j}\right) .
$$

Then,

$$
\begin{aligned}
& \frac{1}{j^{n}} \frac{d^{m+1}}{d \xi^{m+1}}(\xi j+a)_{n} \\
& \quad=m ! \sum_{0 \leq j_{1}<j_{2}<\cdots<j_{n-m} \leq n-1} \frac{d}{d \xi} \prod_{q=1}^{n-m}\left(\xi+\frac{a-j_{q}}{j}\right),
\end{aligned}
$$

where the sum has $\left(\begin{array}{c}n \\ n-m\end{array}\right)=n(n-1)(n-2) \cdots(n-m+1) / m$ ! terms and its summand

$$
\frac{d}{d \xi} \prod_{q=1}^{n-m}\left(\xi+\frac{a-j_{q}}{j}\right)=\sum_{i_{1}<i_{2}<\cdots<i_{n-m-1}} \prod_{q=1}^{n-m-1}\left(\xi+\frac{a-i_{q}}{j}\right),
$$

$i_{q} \in\left\{j_{1}, j_{2}, \ldots, j_{n-m}\right\}$ has $\left(\begin{array}{c}n-m \\ n-m-1\end{array}\right)=(n-m)(n-m-$ $1) ! /(n-m-1) !=n-m$ terms. Therefore, the expansion of $\left(d^{m+1} / d \xi^{m+1}\right)(\xi j+a)_{n}$ has a total of $n(n-1) \cdots(n-$ $m+1)(n-m) / m$ ! terms of the form $\prod_{q=1}^{n-m-1}(\xi+(a-$ $\left.j_{q}\right) / j$ ). However, if the sum is evaluated over all possible combinations $j_{1} j_{2} \cdots j_{n-m-1}$ such that $0 \leq j_{1}<j_{2}<\cdots<$ $j_{n-m-1} \leq n-1$, then the sum has $\left(\begin{array}{c}n \\ n-m-1\end{array}\right)=n(n-1) \cdots(n-$ $m)(n-m-1 !) /(m+1) !(n-m-1) !=(1 /(m+1))(n(n-$ $1) \cdots(n-m) / m$ !) distinct terms. It follows that every term $\prod_{q=1}^{n-m-1}\left(\xi+\left(a-j_{q}\right) / j\right)$ appears $m+1$ times in the expansion of $\left(1 / j^{n}\right)\left(d^{m+1} / d \xi^{m+1}\right)(\xi j+a)_{n}$. Thus we have

$$
\begin{aligned}
& \frac{1}{j^{n}} \frac{d^{m+1}}{d \xi^{m+1}}(\xi j+a)_{n} \\
& \quad=m ! \sum_{0 \leq j_{1}<j_{2}<\cdots<j_{n-m-1} \leq n-1} m+1 \prod_{q=1}^{n-m}\left(\xi+\frac{a-j_{q}}{j}\right) \\
& \quad=(m+1) ! \sum_{0 \leq j_{1}<j_{2}<\cdots<j_{n-m-1} \leq n-1} \prod_{q=1}^{n-m}\left(\xi+\frac{a-j_{q}}{j}\right) .
\end{aligned}
$$

Lemma 2. Let $\widehat{s}(n, k ; a)=$ $(1 / k !) \lim _{\xi \rightarrow 0}\left(\left(\sum_{j=0}^{k}(-1)^{k-j}\left(\begin{array}{c}k \\ j\end{array}\right)\left(d^{k} / d \xi^{k}\right)(\xi j+a)_{n}\right) / k !\right)$. Then

$$
\widehat{s}(n, k ; a)=\sum_{0 \leq j_{1}<j_{2}<\cdots<j_{n-k} \leq n-1} \prod_{q=1}^{n-k}\left(a-j_{q}\right) .
$$

Proof. Using Lemma 1,

$$
\begin{array}{r}
\widehat{\mathcal{s}}(n, k ; a) \\
=\frac{1}{k !} \lim _{\xi \rightarrow 0}\left(\left(\sum_{j=0}^{k}(-1)^{k-j}\left(\begin{array}{c}
k \\
j
\end{array}\right) j^{n} \sum_{0 \leq j_{1}<\cdots<j_{n-k \leq n-1}} k !\right.\right. \\
\left.\left.\times \prod_{q=1}^{n-k}\left(\xi+\frac{a-j_{q}}{j}\right)\right)(k !)^{-1}\right) .
\end{array}
$$


Note that $\prod_{q=1}^{n-k}\left(\xi+\left(a-j_{q}\right) / j\right)=\left(1 / j^{n-k}\right) \prod_{q=1}^{n-k}\left(\xi j+a-j_{q}\right)$. Hence, the expression at the right-hand side of (18) becomes

$$
\begin{aligned}
\frac{1}{k !} \lim _{\xi \rightarrow 0}\left[\sum_{j=0}^{k}(-1)^{k-j}\left(\begin{array}{c}
k \\
j
\end{array}\right) j^{k}\right. & \\
& \left.\times \sum_{0 \leq j_{1}<j_{2}<\cdots<j_{n-k} \leq n-1} \prod_{q=1}^{n-k}\left(\xi j+a-j_{q}\right)\right] \\
=\frac{1}{k !} & {\left[\sum_{j=0}^{k}(-1)^{k-j}\left(\begin{array}{c}
k \\
j
\end{array}\right) j^{k}\right.} \\
& \left.\times \sum_{0 \leq j_{1}<j_{2}<\cdots<j_{n-k} \leq n-1} \prod_{q=1}^{n-k}\left(a-j_{q}\right)\right],
\end{aligned}
$$

which boils down to

$$
\sum_{0 \leq j_{1}<j_{2}<\cdots<j_{n-k} \leq n-1} \prod_{q=1}^{n-k}\left(a-j_{q}\right)
$$

since

$$
\frac{1}{k !} \sum_{j=0}^{k}(-1)^{k-j}\left(\begin{array}{l}
k \\
j
\end{array}\right) j^{k}=S(k, k)=1,
$$

where $S(n, k)$ denote the Stirling numbers of the second kind.

Theorem 3. The noncentral Stirling numbers of the first kind equal

$$
s_{a}(n, k)=\widehat{s}(n, k ; a)=\sum_{0 \leq j_{1}<j_{2}<\cdots<j_{n-k} \leq n-1} \prod_{q=1}^{n-k}\left(a-j_{q}\right) .
$$

Proof. We know that

$$
\sum_{0 \leq j_{1}<j_{2}<\cdots<j_{n-k+1} \leq n} \prod_{q=1}^{n-k+1}\left(a-j_{q}\right)
$$

is equal to the sum of the products $\left(a-j_{1}\right)\left(a-j_{2}\right) \cdots\left(a-j_{n+1-k}\right)$ where the sum is evaluated overall possible combinations $j_{1} j_{2} \cdots j_{n+1-k}, j_{i} \in\{0,1,2, \ldots, n\}$. These possible combinations can be divided into two: the combinations with $j_{i}=n$ for some $i \in\{1,2, \ldots, n-k+1\}$ and the combinations with $j_{i} \neq n$ for all $i \in\{0,1,2, \ldots, n-k+1\}$. Thus

$$
\sum_{0 \leq j_{1}<j_{2}<\cdots<j_{n-k+1} \leq n} \prod_{q=1}^{n-k+1}\left(a-j_{q}\right)
$$

is equal to

$$
\begin{gathered}
\sum_{0 \leq j_{1}<j_{2}<\cdots<j_{n-k+1} \leq n-1} \prod_{q=1}^{n-k+1}\left(a-j_{q}\right)+(a-n) \\
\times \sum_{0 \leq j_{1}<j_{2}<\cdots<j_{n-k} \leq n-1} \prod_{q=1}^{n-k}\left(a-j_{q}\right) .
\end{gathered}
$$

This implies that

$$
\widehat{s}(n+1, k ; a)=\widehat{s}(n, k-1 ; a)+(a-n) \widehat{s}(n, k ; a) .
$$

This is exactly the triangular recurrence relation in (4) for $s_{a}(n, k)$. This proves the theorem.

The explicit formula in Theorem 3 is necessary in locating the peak of the distribution of noncentral Stirling numbers of the first kind. Besides, this explicit formula can also be used to give certain combinatorial interpretation of $s_{a}(n, k)$.

\begin{tabular}{|c|c|c|c|c|}
\hline 0 & 0 & 0 & 1 & 1 \\
\hline 0 & 0 & 1 & & \\
\hline 1 & 0 & 0 & & \\
\hline 0 & 1 & & & \\
\hline 0 & & & & \\
\hline
\end{tabular}

A 0-1 tableau, as defined in [4] by de Médicis and Leroux, is a pair $\varphi=(\lambda, f)$, where

$$
\lambda=\left(\lambda_{1} \geq \lambda_{2} \geq \cdots \geq \lambda_{k}\right)
$$

is a partition of an integer $m$, and $f=\left(f_{i j}\right)_{1 \leq j \leq \lambda_{i}}$ is a "filling" of the cells of corresponding Ferrers diagram of shape $\lambda$ with 0 's and 1's, such that there is exactly one 1 in each column. Using the partition $\lambda=(5,3,3,2,1)$ we can construct 60 distinct 0 1 tableaux. One of these $0-1$ tableaux is given in the following figure with $f_{14}=f_{15}=f_{23}=f_{31}=f_{42}=1, f_{i j}=0$ elsewhere $\left(1 \leq j \leq \lambda_{i}\right)$ :

Also, as defined in [4], an A-tableau is a list $\phi$ of column $c$ of a Ferrers diagram of a partition $\lambda$ (by decreasing order of length) such that the lengths $|c|$ are part of the sequence $A=$ $\left(a_{i}\right)_{i \geq 0}, a_{i} \in Z^{+} \cup\{0\}$. If $\operatorname{Td}^{A}(h, r)$ is the set of $A$-tableaux with exactly $r$ distinct columns whose lengths are in the set $A=$ $\left\{a_{0}, a_{1}, \ldots, a_{h}\right\}$, then $\left|T d^{A}(h, r)\right|=\left(\begin{array}{c}h+1 \\ r\end{array}\right)$. Now, transforming each column $c$ of an $A$-tableau in $\operatorname{Td}^{A}(n-1, n-k)$ into a column of length $\omega(|c|)$, we obtain a new tableau which is called $A_{\omega}$-tableau. If $\omega(|c|)=|c|$, then the $A_{\omega}$-tableau is simply the $A$-tableau. Now, we define an $A_{\omega}(0,1)$-tableau to be a $0-1$ tableau which is constructed by filling up the cells of an $A_{\omega}$-tableau with 0 's and 1's such that there is only one 1 in each column. We use $T d^{A_{\omega}(0,1)}(n-1, n-k)$ to denote the set of such $A_{\omega}(0,1)$-tableaux.

It can easily be seen that every $(n-k)$ combination $j_{1} j_{2} \cdots j_{n-k}$ of the set $\{0,1,2, \ldots, n-1\}$ can be represented geometrically by an element $\phi$ in $T d^{A}(n-1, n-k)$ with $j_{i}$ as the length of $(n-k-i+1)$ th column of $\phi$ where $A=$ $\{0,1,2, \ldots, n-1\}$. Hence, with $\omega(|c|)=a-|c|,(22)$ may be written as

$$
s_{a}(n, k)=\sum_{\phi \in T d^{A}(n-1, n-k)} \prod_{c \in \phi} \omega(|c|) .
$$

Thus, using (29), we can easily prove the following theorem.

Theorem 4. The number of $A_{\omega}(0,1)$-tableaux in $\operatorname{Td}^{A_{\omega}(0,1)}(n-$ $1, n-k)$ where $A=\{0,1,2, \ldots, n-1\}$ such that $\omega(|c|)=a-|c|$ is equal to $s_{a}(n, k)$. 
Let $\phi$ be an $A$-tableau in $T d^{A}(n-1, n-k)$ with $A=$ $\{0,1,2, \ldots, n-1\}$, and

$$
\begin{aligned}
\omega_{A}(\phi) & =\prod_{c \in \phi} \omega(|c|) \\
& =\prod_{i=1}^{n-k}\left(a-j_{i}\right), \quad j_{i} \in\{0,1,2, \ldots, n-1\} .
\end{aligned}
$$

If $a=a_{1}+a_{2}$ for some $a_{1}$ and $a_{2}$, then, with $\omega^{*}(j)=a_{2}-j$,

$$
\begin{aligned}
\omega_{A}(\phi) & =\prod_{i=1}^{n-k}\left(a_{1}+\omega^{*}\left(j_{i}\right)\right) \\
& =\sum_{r=0}^{n-k} a_{1}^{n-k-r} \sum_{j_{i} \leq q_{1}<q_{2}<\cdots<q_{r} \leq j_{n-k}} \prod_{i=1}^{r} \omega^{*}\left(q_{i}\right) .
\end{aligned}
$$

Suppose $B_{\phi}$ is the set of all $A$-tableaux corresponding to $\phi$ such that for each $\psi \in B_{\phi}$ either

$\psi$ has no column whose weight is $a_{1}$, or

$\psi$ has one column whose weight is $a_{1}$, or

$\psi$ has $n-k$ columns whose weights are $a_{1}$. Then, we may write

$$
\omega_{A}(\phi)=\sum_{\psi \in B_{\phi}} \omega_{A}(\psi) .
$$

Now, if $r$ columns in $\psi$ have weights other than $a_{1}$, then

$$
\omega_{A}(\psi)=a_{1}^{n-k-r} \prod_{i=1}^{r} \omega^{*}\left(q_{i}\right),
$$

where $q_{1}, q_{2}, \ldots, q_{r} \in\left\{j_{1}, j_{2}, \ldots, j_{n-k}\right\}$. Hence, (29) may be written as

$$
s_{a}(n, k)=\sum_{\phi \in T d^{A}(n-1, n-k)} \sum_{\psi \in B_{\phi}} \omega_{A}(\psi) .
$$

Note that for each $r$, there correspond $\left(\begin{array}{c}n-k \\ r\end{array}\right)$ tableaux with $r$ distinct columns having weights $w^{*}\left(q_{i}\right), q_{i} \in\left\{j_{1}, j_{2}, \ldots, j_{n-k}\right\}$. Since $T d^{A}(n-1, n-k)$ has $\left(\begin{array}{l}n \\ k\end{array}\right)$ elements, for each $\phi \in \operatorname{Td}^{A}(n-$ $1, n-k)$, the total number of $A$-tableaux $\psi$ corresponding to $\phi$ is

$$
\left(\begin{array}{l}
n \\
k
\end{array}\right)\left(\begin{array}{c}
n-k \\
r
\end{array}\right)
$$

elements. However, only $\left(\begin{array}{c}n \\ r\end{array}\right)$ tableaux in $B_{\phi}$ with $r$ distinct columns of weights other than $a_{1}$ are distinct. Hence, every distinct tableau $\psi$ appears

$$
\frac{\left(\begin{array}{c}
n \\
k
\end{array}\right)\left(\begin{array}{c}
n-k \\
r
\end{array}\right)}{\left(\begin{array}{c}
n \\
r
\end{array}\right)}=\left(\begin{array}{c}
n-r \\
k
\end{array}\right)
$$

times in the collection. Consequently, we obtain

$$
s_{a}(n, k)=\sum_{r=0}^{n-k}\left(\begin{array}{c}
n-r \\
k
\end{array}\right) a_{1}^{n-k-r} \sum_{\psi \in B_{r}} \prod_{c \in \psi} \omega^{*}(|c|),
$$

where $B_{r}$ denotes the set of all tableaux $\psi$ having $r$ distinct columns whose lengths are in the set $\{0,1,2, \ldots, n-1\}$. Reindexing the double sum, we get

$$
s_{a}(n, k)=\sum_{j=k}^{n}\left(\begin{array}{l}
j \\
k
\end{array}\right) a_{1}^{j-k} \sum_{\psi^{*} \in B_{n-j}} \prod_{c \in \psi^{*}} \omega^{*}(|c|) .
$$

Clearly, $B_{n-j}=\operatorname{Td}^{A}(n-1, n-j)$. Thus, using (22), we obtain the following theorem.

Theorem 5. The numbers $s_{a}(n, k)$ satisfy the following identity:

$$
s_{a}(n, k)=\sum_{j=k}^{n}\left(\begin{array}{l}
j \\
k
\end{array}\right) a_{1}^{j-k} s_{a_{2}}(n, j),
$$

where $a=a_{1}+a_{2}$ for some numbers $a_{1}$ and $a_{2}$.

The next theorem contains certain convolution-type formula for $s_{a}(n, k)$ which will be proved using the combinatorics of $A$-tableau.

Theorem 6. The numbers $s_{a}(n, k)$ have convolution formula

$$
s_{a}(m+j, n)=\sum_{k=0}^{n} s_{a}(m, k) s_{a-m}(j, n-k) .
$$

Proof. Suppose that $\phi_{1}$ is a tableau with exactly $m-k$ distinct columns whose lengths are in the set $A_{1}=\{0,1,2, \ldots, m-1\}$ and $\phi_{2}$ is a tableau with exactly $j-n+k$ distinct columns whose lengths are in the set $A_{2}=\{m, m+1, m+2, \ldots, m+j-1\}$. Then $\phi_{1} \in \operatorname{Td}^{A_{1}}(m-1, m-k)$ and $\phi_{2} \in T d^{A_{2}}(j-1, j-n+k)$. Notice that by joining the columns of $\phi_{1}$ and $\phi_{2}$, we obtain an $A$-tableau $\phi$ with $m+j-n$ distinct columns whose lengths are in the set $A=\{0,1,2, \ldots, m+j-1\}$; that is, $\phi \in \operatorname{Td}^{A}(m+$ $j-1, m+j-n)$. Hence

$$
\begin{gathered}
\sum_{\phi \in T d^{A}(m+j-1, m+j-n)} \omega_{A}(\phi) \\
=\sum_{k=0}^{n}\left\{\sum_{\phi_{1} \in T d^{A_{1}}(m-1, m-k)} \omega_{A_{1}}\left(\phi_{1}\right)\right\} \\
\times\left\{\sum_{\phi_{2} \in T d^{A_{2}}(j-1, j-n+k)} \omega_{A_{2}}\left(\phi_{2}\right)\right\} .
\end{gathered}
$$


Note that

$$
\begin{aligned}
& \sum_{\phi_{2} \in T d^{A_{2}}} \omega_{(j-1, j-n+k)}\left(\phi_{2}\right) \\
= & \sum_{m \leq g_{1}<g_{2}<\cdots<g_{j-n+k} \leq m+j-1} \prod_{q=1}^{j-n+k}\left(a-g_{q}\right) \\
= & \sum_{0 \leq g_{1}<g_{2}<\cdots<g_{n-k-j} \leq j-1} \prod_{q=1}^{j-n+k}\left(a-\left(m+g_{q}\right)\right) \\
= & s_{a-m}(j, n-k) .
\end{aligned}
$$

Also, using (29), we have

$$
\begin{aligned}
\sum_{\phi_{1} \in T d^{A_{1}}(m-1, m-k)} \omega_{A_{1}}\left(\phi_{1}\right) & =s_{a}(m, k) \\
\sum_{\phi \in T d^{A}(m+j-1, m+j-n)} \omega_{A}(\phi) & =s_{a}(m+j, n) .
\end{aligned}
$$

Thus,

$$
s_{a}(m+j, n)=\sum_{k=0}^{n} s_{a}(m, k) s_{a-m}(j, n-k) .
$$
formula.

The following theorem gives another form of convolution

Theorem 7. The numbers $s_{a}(n, k)$ satisfy the second form of convolution formula

$$
s_{a}(n+1, m+j+1)=\sum_{k=0}^{n} s_{a}(k, n) s_{a-(k+1)}(n-k, j) .
$$

Proof. Let

$\phi_{1}$ be a tableau with $k-m$ columns whose lengths are in $A_{1}=\{0,1, \ldots, k-1\}$,

$\phi_{2}$ be a tableau with $n-k-j$ columns whose lengths are in $A_{2}=\{k+1, \ldots, n\}$.

Then $\phi_{1} \in T d^{A_{1}}(k-1, k-m) ; \phi_{2} \in \operatorname{Td}^{A_{2}}(n-k-1, n-k-$ $j)$. Using the same argument above, we can easily obtain the convolution formula.

\section{The Maximum of Noncentral Stirling Numbers of the First Kind}

We are now ready to locate the maximum of $s_{a}(n, k)$. First, let us consider the following theorem on Newton's inequality [5] which is a good tool in proving log-concavity or unimodality of certain combinatorial sequences.

Theorem 8. If the polynomial $a_{1} x+a_{2} x^{2}+\cdots+a_{n} x^{n}$ has only real zeros then

$$
a_{k}^{2} \geq a_{k+1} a_{k-1} \frac{k}{k-1} \frac{n-k+1}{n-k} \quad(k=2, \ldots, n-1) .
$$

Now, consider the following polynomial:

$$
\sum_{k=0}^{n} s_{a}(n, k)(t+a)^{k}
$$

This polynomial is just the expansion of the factorial $\langle t\rangle_{n}=t(t+1)(t+2) \cdots(t+n-1)$ which has real roots $0,-1,-2, \ldots,-n+1$. If we replace $t$ by $t-a$, we see at once that the roots of the polynomial $\sum_{k=0}^{n} s_{a}(n, k) t^{k}$ are $a, a-1, \ldots, a-$ $n+1$. Applying Newton's Inequality completes the proof of the following theorem.

Theorem 9. The sequence $\left\{s_{a}(n, k)\right\}_{k=0}^{n}$ is strictly log-concave and, hence, unimodal.

By replacing $t$ with $-t$, the relation in (1) may be written as

$$
\langle t\rangle_{n}=\sum_{k=0}^{n}(-1)^{n-k} s_{a}(n, k)(t+a)^{k}
$$

where $\langle t\rangle_{n}=t(t+1)(t+2) \cdots(t+n-1)$. Note that, from Theorem 3 with $a<0$,

$$
s_{a}(n, k)=(-1)^{n-k} \sum_{0 \leq j_{1}<j_{2}<\cdots<j_{n-k} \leq n-1} \prod_{q=1}^{n-k}\left(b+j_{q}\right),
$$

where $b=-a>0$. Now, we define the signless noncentral Stirling number of the first kind, denoted by $\left|s_{a}(n, k)\right|$, as

$$
\begin{aligned}
\left|s_{a}(n, k)\right| & =(-1)^{n-k} s_{a}(n, k) \\
& =\sum_{0 \leq j_{1}<j_{2}<\cdots<j_{n-k} \leq n-1} \prod_{q=1}^{n-k}\left(b+j_{q}\right) .
\end{aligned}
$$

To introduce the main result of this paper, we need to state first the following theorem of Erdös and Stone [3].

Theorem 10 (see [3]). Let $u_{1}<u_{2}<\cdots$ be an infinite sequence of positive real numbers such that

$$
\sum_{i=1}^{\infty} \frac{1}{u_{i}}=\infty, \quad \sum_{i=1}^{\infty} \frac{1}{u_{i}^{2}}<\infty .
$$

Denote by $\sum_{n, k}$ the sum of the product of the first $n$ of them taken $k$ at a time and denote by $K_{n}$ the largest value of $k$ for which $\sum_{n, k}$ assumes its maximum value. Then

$$
K_{n}=n-\left[\sum_{i=1}^{n} \frac{1}{u_{i}}-\sum_{i=1}^{n} \frac{1}{u_{i}^{2}}\left(1+\frac{1}{u_{i}}\right)^{-1}+o(1)\right] .
$$

We also need to recall the asymptotic expansion of harmonic numbers which is given by

$$
\frac{1}{1}+\frac{1}{2}+\cdots+\frac{1}{n}=\log n+\gamma+o(1)
$$

where $\gamma$ is the Euler-Mascheroni constant.

The following theorem contains a formula that determines the value of the index corresponding to the maximum of the sequence $\left\{\left|s_{a}(n, k)\right|\right\}_{k=0}^{n}$. 
Theorem 11. The largest index for which the sequence $\left\{\left|s_{a}(n, k)\right|\right\}_{k=0}^{n}$ assumes its maximum is given by the approximation

$$
k_{a, n}=\left[\log \left(\frac{b+n}{b}\right)+o(1)\right]
$$

where $[x]$ is the integer part of $x$ and $b=-a, a<0$.

Proof. Using Theorem 10 and by (50), we see that $\left|s_{a}(n, k)\right|=$ $\sum_{n-1, n-k}$. Denoting by $k_{a, n}$ for which $\sum_{n, n-k}$ is maximum and with $u_{1}=b+0, u_{2}=b+1, \ldots, u_{n-1}=b+n-1$ we have

$$
\begin{aligned}
k_{a, n} & =\left[\sum_{i=0}^{n-1} \frac{1}{b+i}-\sum_{i=0}^{n-1} \frac{1}{(b+i)^{2}}\left(1+\frac{1}{b+i}\right)^{-1}+o(1)\right] \\
& =\left[\sum_{i=0}^{n-1} \frac{1}{b+i}-\sum_{i=0}^{n-1} \frac{1}{(b+i)(b+i+1)}+o(1)\right] \\
& =\left[\sum_{i=0}^{n-1} \frac{1}{b+i+1}+o(1)\right] .
\end{aligned}
$$

But using (53), we see that

$$
\sum_{i=0}^{n-1} \frac{1}{b+i+1}=\log (b+n)-\log b .
$$

From this we get

$$
k_{a, n}=\left[\log \left(\frac{b+n}{b}\right)+o(1)\right] .
$$

For the case in which $a>0$ we will only consider the sequence of noncentral Stirling numbers of the first kind for which $a \geq n$.

Theorem 12. The maximizing index for which the maximum noncentral Stirling number occurs for $a \geq n$ is given by the approximation

$$
k_{a, n}=\left[\log \left(\frac{a+1}{a-n+1}\right)+o(1)\right] .
$$

Proof. From the definition, for $a \geq n, s_{a}(n, k)>0$ and by Theorem $3, s_{a}(n, k)$ is the sum of the products $\left(a-j_{1}\right)(a-$ $\left.j_{2}\right) \cdots\left(a-j_{n-k}\right)$ where $j_{i}$ 's are taken from the set $\{0,1,2, \ldots, n-$ $1\}$. By Theorem 10, $s_{a}(n, k)=\sum_{n, n-k}$. Thus with $u_{1}=a, u_{2}=$ $a-1, \ldots, u_{n-1}=a-n+1$ we have

$$
\begin{aligned}
k_{a, n} & =\left[\sum_{i=0}^{n-1} \frac{1}{a-i}-\sum_{i=0}^{n-1} \frac{1}{(a-i)^{2}}\left(1+\frac{1}{a-i}\right)^{-1}+o(1)\right] \\
& =\left[\sum_{i=0}^{n-1} \frac{1}{a-i}-\sum_{i=0}^{n-1} \frac{1}{(a-i)(a-i+1)}+o(1)\right] \\
& =\left[\sum_{i=0}^{n-1} \frac{1}{a-i+1}+o(1)\right] .
\end{aligned}
$$

TABLE 1: Values of $s_{-1}(n, k)$.

\begin{tabular}{lcccc}
\hline$n / k$ & 0 & 1 & 2 & 3 \\
\hline 0 & 1 & & & \\
1 & 1 & & & \\
2 & 2 & 1 & & \\
3 & 6 & 6 & 1 & \\
4 & 24 & 35 & 10 & 1 \\
5 & 120 & 225 & 85 & 15 \\
6 & 720 & 1624 & 735 & 175 \\
7 & 5040 & 13132 & 6769 & 1960 \\
8 & 40320 & 109584 & 118124 & 67284 \\
9 & 362880 & 1026576 & 1172700 & 723680 \\
10 & 3628800 & 10628640 & 12753576 & 8409500 \\
\hline
\end{tabular}

Again, using (53), we get

$$
k_{a, n}=\left[\log \left(\frac{a+1}{a-n+1}\right)+o(1)\right] .
$$

Example 13. The maximum element of the sequence $\left\{s_{-1}(9, k)\right\}_{k=0}^{9}$ occurs at (Table 1$)$

$$
\begin{aligned}
k_{-1,9} & =\left[\log \left(\frac{1+8}{1}\right)+o(1)\right] \\
& =[\log 9+o(1)] \\
& \approx 2 .
\end{aligned}
$$

Example 14. The maximum element of the sequence $\left\{s_{10}(10, k)\right\}_{k=0}^{10}$ occurs at (Table 2$)$

$$
\begin{aligned}
k_{10,10} & =\left[\log \left(\frac{10+1}{10-10+1}\right)+o(1)\right] \\
& =[\log 11+o(1)] \\
& \approx 2 .
\end{aligned}
$$

We know that the classical Stirling numbers of the first kind are special cases of $s_{a}(n, k)$ by taking $a=0$. However, formulas in Theorems 11 and 12 do not hold when $a=0$. Hence, these formulas are not applicable to determine the maximum of the classical Stirling numbers. Here, we derive a formula that determines the value of the index corresponding to the maximum of the signless Stirling numbers of the first kind.

The signless Stirling numbers of the first kind [6] are the sum of all products of $n-k$ different integers taken from $\{1,2,3, \ldots, n-1\}$. That is,

$$
|s(n, k)|=\sum_{1 \leq i_{1}<i_{2}<\cdots<i_{n-k} \leq n-1} i_{1} i_{2} \cdots i_{n-k}
$$


TABLE 2: Values of $s_{10}(n, k)$.

\begin{tabular}{lcccc}
\hline$n / k$ & 0 & 1 & 2 & 3 \\
\hline 0 & 1 & & & \\
1 & 10 & 1 & & \\
2 & 90 & 19 & 1 & \\
3 & 720 & 242 & 27 & 1 \\
4 & 5040 & 2414 & 431 & 34 \\
5 & 30240 & 19524 & 5000 & 635 \\
6 & 151200 & 127860 & 44524 & 8175 \\
7 & 604800 & 662640 & 305956 & 77224 \\
8 & 1814400 & 2592720 & 1580508 & 537628 \\
9 & 3628800 & 6999840 & 5753736 & 2655764 \\
10 & 3628800 & 10628640 & 12753576 & 8409500 \\
\hline
\end{tabular}

TABLE 3: Values of $|s(n, k)|$ for $0 \leq n \leq 10$.

\begin{tabular}{lcccccc}
\hline$s(n, k)$ & 0 & 1 & 2 & 3 & 4 & 5 \\
\hline 0 & 1 & & & & & \\
1 & 0 & 1 & & & & \\
2 & 0 & 1 & 1 & & & \\
3 & 0 & 2 & 3 & 1 & & \\
4 & 0 & 6 & 11 & 6 & 1 & \\
5 & 0 & 24 & 50 & 35 & 10 & 1 \\
6 & 0 & 120 & 274 & 225 & 85 & 15 \\
7 & 0 & 720 & 1764 & 1624 & 735 & 175 \\
8 & 0 & 540 & 13068 & 13132 & 6769 & 1960 \\
9 & 0 & 40320 & 109584 & 118124 & 67284 & 22449 \\
10 & 0 & 362880 & 1026576 & 1172700 & 723680 & 269325 \\
\hline
\end{tabular}

Using Theorem 10, $|s(n, k)|=\sum_{n-1, n-k}$. We use $k_{n}$ to denote the largest value of $k$ for which $\sum_{n-1, n-k}$ is maximum. With $u_{1}=1, u_{2}=2, \ldots, u_{n-1}=n-1$ we have

$$
\begin{aligned}
k_{n} & =1+\left[\sum_{i=1}^{n-1} \frac{1}{i}-\sum_{i=1}^{n-1} \frac{1}{i^{2}}\left(1+\frac{1}{i}\right)^{-1}+o(1)\right] \\
& =1+\left[\sum_{i=1}^{n-1} \frac{1}{i}-\sum_{i=1}^{n-1}\left(\frac{1}{i}-\frac{1}{i+1}\right)+o(1)\right] \\
& =1+\left[\sum_{i=1}^{n-1} \frac{1}{i+1}+o(1)\right] .
\end{aligned}
$$

Using (53), we see that

$$
\sum_{i=1}^{n-1} \frac{1}{1+i}=\log n-\log 1+\gamma+o(1)
$$

Therefore, we have

$$
k_{n}=[\log n+\gamma+o(1)] .
$$

Example 15. It is shown in Table 3 that the maximum value of $|s(n, k)|$ when $n=7$ occurs at $k=2$. Using (66), it can be verified that the maximum element of the sequence $\{|s(7, k)|\}_{k=0}^{7}$ occurs at

$$
\begin{aligned}
k_{7} & =[\log 7+\gamma+o(1)] \\
& =[1.95+0.5772+o(1)] \\
& =[2.53+o(1)] \\
& \approx 2 .
\end{aligned}
$$

Moreover, when $n=10$, the maximum value occurs at

$$
\begin{aligned}
k_{10} & =[\log 10+\gamma+o(1)] \\
& =[2.30+0.5772+o(1)] \\
& =[2.8772+o(1)] \\
& \approx 3 .
\end{aligned}
$$

Recently, a paper by Cakić et al. [7] established explicit formulas for multiparameter noncentral Stirling numbers which are expressible in symmetric function forms. One may then try to investigate the location of the maximum value of these numbers using the Erdös-Stone theorem.

\section{Conflict of Interests}

The authors declare that there is no conflict of interests regarding the publication of this paper.

\section{Acknowledgment}

The authors wish to thank the referees for reading the paper thoroughly.

\section{References}

[1] M. Koutras, "Noncentral Stirling numbers and some applications," Discrete Mathematics, vol. 42, no. 1, pp. 73-89, 1982.

[2] I. Mezö, "On the maximum of $r$-Stirling numbers," Advances in Applied Mathematics, vol. 41, no. 3, pp. 293-306, 2008.

[3] P. Erdös, "On a conjecture of Hammersley," Journal of the London Mathematical Society, vol. 28, pp. 232-236, 1953.

[4] A. de Médicis and P. Leroux, "Generalized Stirling numbers, convolution formulae and $p, q$-analogues," Canadian Journal of Mathematics, vol. 47, no. 3, pp. 474-499, 1995.

[5] E. H. Lieb, "Concavity properties and a generating function for Stirling numbers," Journal of Combinatorial Theory, vol. 5, no. 2, pp. 203-206, 1968.

[6] L. Comtet, Advanced Combinatorics, Reidel, Dordrecht, The Netherlands, 1974.

[7] N. P. Cakić, B. S. El-Desouky, and G. V. Milovanović, "Explicit formulas and combinatorial identities for generalized Stirling numbers," Mediterranean Journal of Mathematics, vol. 10, no. 1, pp. 57-72, 2013. 


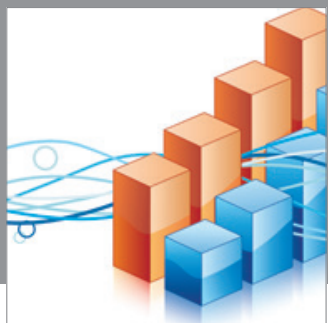

Advances in

Operations Research

mansans

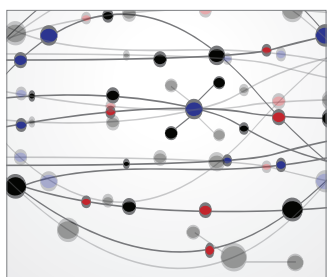

The Scientific World Journal
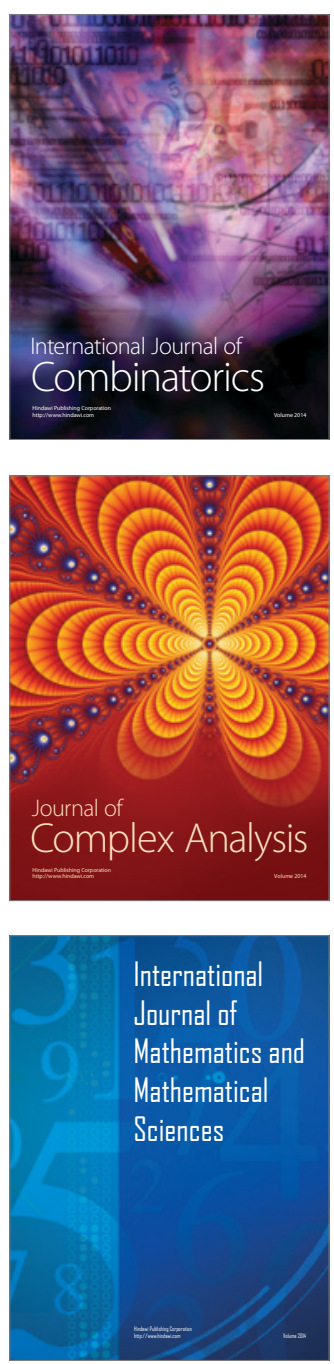
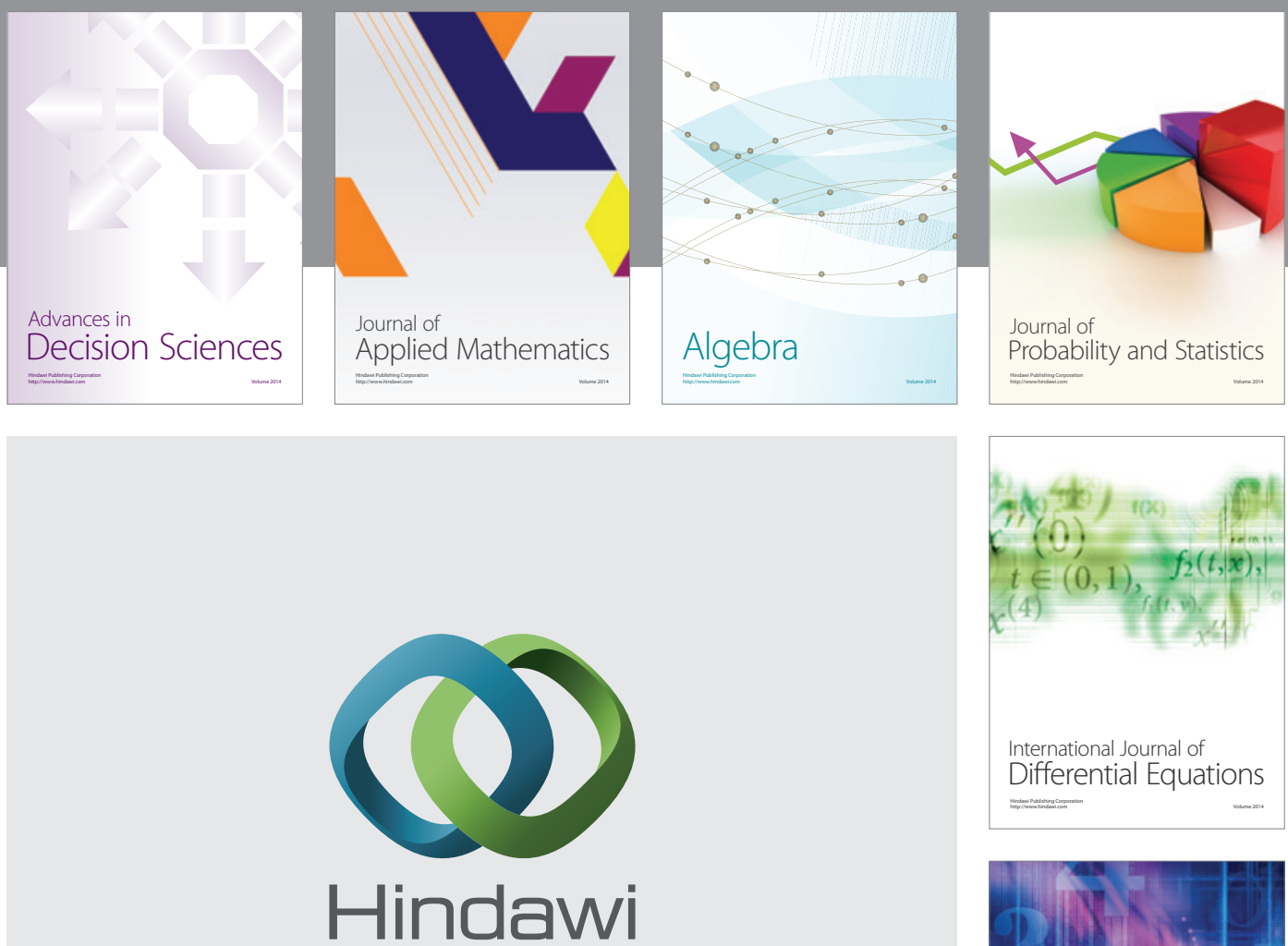

Submit your manuscripts at http://www.hindawi.com
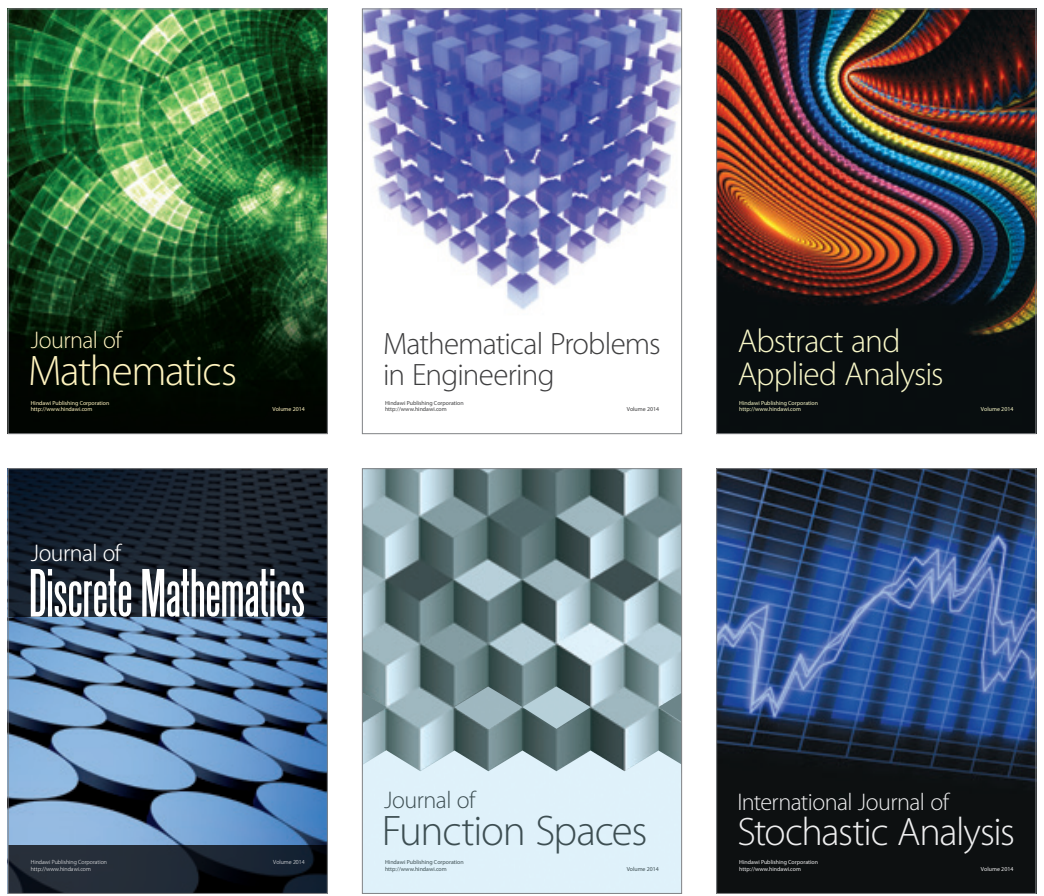

Journal of

Function Spaces

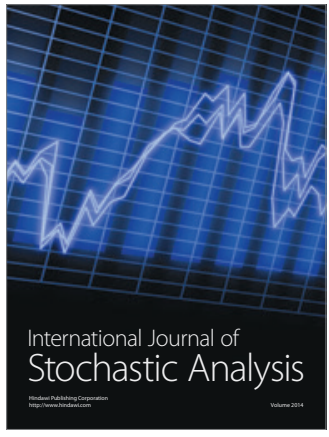

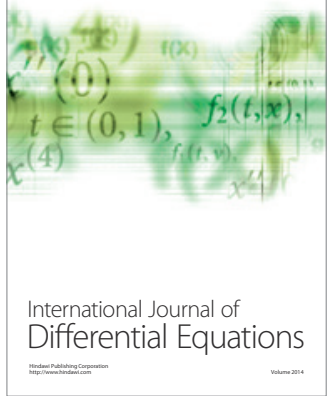
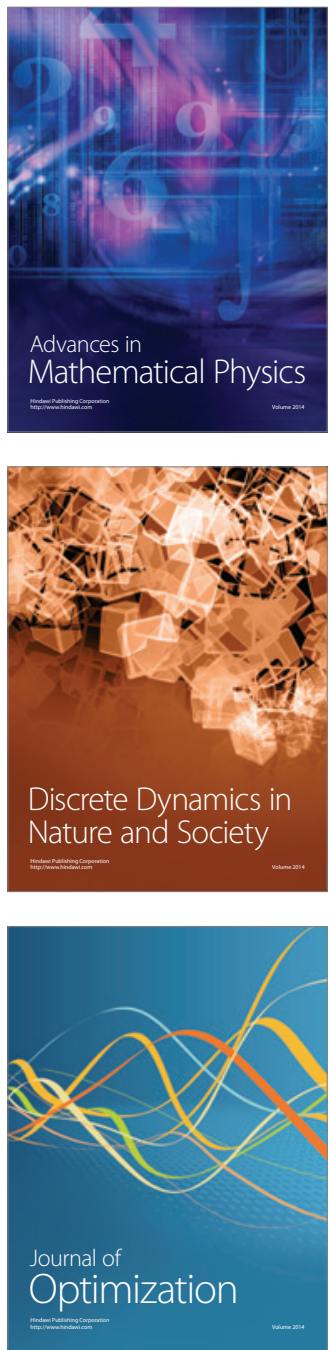